\title{
CD122 blockade restores immunological tolerance in autoimmune type 1 diabetes via multiple mechanisms
}

\author{
Xiaomei Yuan, ${ }^{1}$ Yi Dong, ${ }^{1}$ Naoya Tsurushita, ${ }^{2}$ J. Yun Tso, ${ }^{2}$ and Wenxian Fu ${ }^{1,3,4}$ \\ 'Pediatric Diabetes Research Center, Department of Pediatrics, UCSD, La Jolla, California, USA. ${ }^{2} J N$ Biosciences, \\ Mountain View, California, USA. ${ }^{3}$ nnstitute for Diabetes and Metabolic Health and ${ }^{4}$ Moores Cancer Center, UCSD, \\ La Jolla, California, USA.
}

Signaling through IL-2/IL-15Rß (CD122) is essential for the differentiation and function of T cells and NK cells. A mAb against CD122 has been implicated to suppress autoimmune type 1 diabetes (T1D) development in animal models. However, the mechanisms remain poorly understood. We find that in vivo administration of an anti-CD122 mAb (CD122 blockade) restores immune tolerance in nonobese diabetic (NOD) mice via multiple mechanisms. First, CD122 blockade selectively ablates pathogenic NK cells and memory phenotype CD8 ${ }^{+} \mathrm{T}$ cells from pancreatic islets. In contrast, islet CD4+Foxp3 ${ }^{+}$Tregs are only mildly affected. Second, CD122 blockade suppresses IFN- $\gamma$ production in islet immune cells. Third, CD122 blockade inhibits the conversion of islet Th17 cells into diabetogenic Th1 cells. Furthermore, a combination of anti-CD122 mAb and Treg-trophic cytokines (IL-2 or IL-33) enhances the abundance and function of islet Tregs. In summary, these data provide crucial mechanistic insights into CD122 blockade-mediated immunoregulation and support therapeutic benefits of this combinational treatment in T1D.

Conflict of interest: NT and JYT are managing partners of JN Biosciences LLC.

Submitted: August 8, 2017 Accepted: December 12, 2017 Published: January 25, 2018

\section{Reference information:} JCI Insight. 2018;3(2):e96600. https://doi.org/10.1172/jici. insight. 96600 .

\section{Introduction}

Type 1 diabetes (T1D) is a prototypical autoimmune disease, characterized by specific destruction of insulin-producing $\beta$ cells by a mistakenly activated self-immune system $(1,2)$. T1D unfolds in a chronic manner and is preceded by chronic islet-specific inflammation (insulitis). During the development of insulitis, a variety of immune populations - including T cells, NK cells, and myeloid cells — infiltrate into the islets of Langerhans in the pancreas $(3,4)$. The roles of these immune cells in T1D vary from disease promoting to disease suppressing $(4,5)$. The aggressiveness of insulitis is determined by the balance between pathogenic and regulatory factors, on both cellular and molecular levels (6). In other words, immune dysregulations break the tolerance to self and facilitate the progression from insulitic to diabetic phase. An approach that can reset the balance between pathogenic and regulatory components of insulitis is considered to have therapeutic benefits for $\operatorname{T1D}(6,7)$.

T cells $(3,8)$ and NK cells $(9,10)$ are key components of T1D-associated insulitis. IL-2 and IL-15 $(11,12)$ are 2 crucial cytokines for the expansion and survival of T cells and NK cells. IL-2/IL-15R $\beta$ (also known as CD122) is an integral part of the receptor complex for both IL-2 and IL-15 (12). It thus can be speculated that modulating CD122 signaling may impact the activities of all CD122-bearing cells in normal and diseased conditions. Indeed, a mAb against CD122 (clone TM- $\beta 1$, rat IgG2b) has been reported to suppress diabetes development in nonobese diabetic (NOD) mice (13-16), suggesting a therapeutic potential of CD122 blockade for human T1D patients. However, the underlying mechanisms remain largely undefined.

In this study, we define the mechanisms by which CD122 blockade prevents T1D development. Using the NOD mouse strain, the primary animal model for T1D basic research and preclinical studies $(8,17)$, we showed that a Fc-silent rat/mouse chimeric anti-CD122 mAb (clone ChMBC7) effectively suppressed T1D development, consistent with previous reports (13-16). Using this engineered anti-CD122 mAb to modulate IL-2/IL-15R $\beta$ signaling, we have investigated how T1D-associated autoimmunity at a prediabetic stage was regulated. Our studies demonstrated that CD122 blockade preferentially affected major populations of islet-associated pathogenic cells by reducing their abundance and suppressing their differentiation into diabetogenic cells. In contrast, we found that Tregs 
were only mildly affected by CD122 blockade. Therefore, these findings support a potentially novel model of restoring immune tolerance in T1D. Furthermore, we also examined the effects of a combinational regimen of anti-CD122 and Treg-trophic cytokines on boosting Treg abundance and function.

\section{Results}

CD122 blockade suppresses insulitis and diabetes in NOD mice. Several studies have reported that an in vivo treatment with an anti-mouse CD122 mAb (clone TM- $\beta 1$, rat IgG2b) (18) prevented the development of diabetes in NOD mice (13-16). However, the underlying mechanisms remain poorly defined. In this study, we have attempted to explore how anti-CD122 suppressed T1D in animal models. To eliminate Fc receptor-mediated (FcR-mediated) effector function, we have developed an Fc-silent, chimeric rat/mouse $\mathrm{mAb}$ for CD122 (clone ChMBC7). ChMBC7 and TM- $\beta 1$ bind to the same epitope of mouse CD122 (Supplemental Figure 1; supplemental material available online with this article; https://doi.org/10.1172/jci. insight.96600DS1) and exhibited a similar potency in inhibiting IL-15 transpresentation (Supplemental Figure 2) (see Method section for more details about the development of ChMBC7 and the comparison with TM- $\beta 1$ ). We next validated the effect of ChMBC7 on the depletion of CD122+ cells (e.g., blood NK cells). In this regard, 10-week-old prediabetic NOD mice were randomly grouped and i.p. injected with 1 dose of ChMBC7 or TM- $\beta 1$ as a control. We analyzed the ablation and recovery of blood CD $122^{+} \mathrm{NK}$ cells longitudinally over a 4 -week period at multiple time points. ChMBC7 injection induced NK depletion, exhibiting deferred kinetics, compared with that by TM- $\beta 1$ (Figure 1A). Moreover, the effect of ChMBC7 in NK ablation lasted longer than that of TM- $\beta 1$, indicating an extended serum half-life. After 4 weeks, the abundance of blood NK cells recovered to $50 \%$ of a basal level in both ChMBC7- and TM- $\beta 1$-treated mice. Together, these data suggest that ChMBC7, an Fc-silent form of anti-CD122 mAb, can impact CD122+ cells, comparable with TM- $\beta 1$.

We next validated the effect of ChMBC7 on T1D development. In this regard, randomly grouped female NOD mice were treated with ChMBC7, or isotype control mAb, twice a week from 3-10 weeks of age. After the treatment, all mice were monitored for spontaneous development of diabetes until 40 weeks of age. The incidence of diabetes onset in ChMBC7-treated mice was significantly lower than that in the control group (Figure 1B), consistent with previous reports (13-16). Using the same treatment protocol, separated cohorts of mice were sacrificed at 10 weeks old, and the pancreata were excised and processed for histopathology analysis. As expected, there was a substantial degree of insulitis in the pancreas of control mice at this age. In contrast, the severity of insulitis was markedly reduced in ChMBC7-treated mice (Figure 1C). ChMBC7-mediated insulitis suppression was further confirmed by comparing the total numbers of pancreas-infiltrated CD45 ${ }^{+}$immune cells from ChMBC7 or control mAb-treated mice (Figure 1D). Therefore, in vivo CD122 blockade by ChMBC7 suppresses insulitis and prevents diabetes development in NOD mice.

CD122 is abundantly expressed in pancreatic NK cells and memory phenotype T cells. Next, we focused on elucidating the mechanisms by which CD122 blockade suppressed T1D. To define what cells were primarily affected by ChMBC7, we first examined the expression of CD122 across various types of immune cells using multiple methods. First, by querying the publicly available Immunological Genome database (www. ImmGen.org) (19), we examined the expression of $C d 122$ on a transcriptional level to define which immune cells express $C d 122$. Clearly, the expression of $C d 122$ was restricted to lineages of NK cells and T cells (both $\mathrm{TCR} \alpha \beta^{+}$and $\mathrm{TCR} \gamma \delta^{+}$), though variations were found within different subsets (Figure 2A). Cd122 transcript was also abundantly detected in Foxp $3^{+}$Tregs (Figure 2A).

T1D is associated with a tissue-specific (pancreatic islet-specific) inflammation characterized by the infiltration of a variety of immune cells, including $\mathrm{T}$ cells and NK cells $(2,3)$. However, the expression of CD122 in different immune populations from T1D-associated pathological lesions remains undefined. We analyzed CD122 expression on a protein level in immune cells isolated from pancreatic islets, pancreas-draining lymph nodes (panLNs), and spleen. Enzymatic digestions used to isolate immune cells from pancreatic islets did not affect the detection of CD122 expression by flow cytometry (Supplemental Figure 3). Our analyses revealed both similarities and differences of CD122 expression between lymphoid organs and pancreatic islets. In all 3 tissues examined (spleen, panLNs, and pancreatic islets), CD122 was most abundantly expressed in NK and NKT cells (Figure $2 \mathrm{~B})$. T cells $\left(\mathrm{CD} 8^{+} \mathrm{T}\right.$ cells and $\mathrm{CD} 4^{+} \mathrm{T}$ cells, including Tregs) were also positive for CD122 expression in all tissues, albeit at a relatively lower level, compared with NK and NKT cells (Figure 2B). Interestingly, the expression of CD122 in TCR $\gamma \delta^{+} \mathrm{T}$ cells was only detected in lymphoid organs - not at tissue sites. We next examined the expression of CD122 in fractioned 

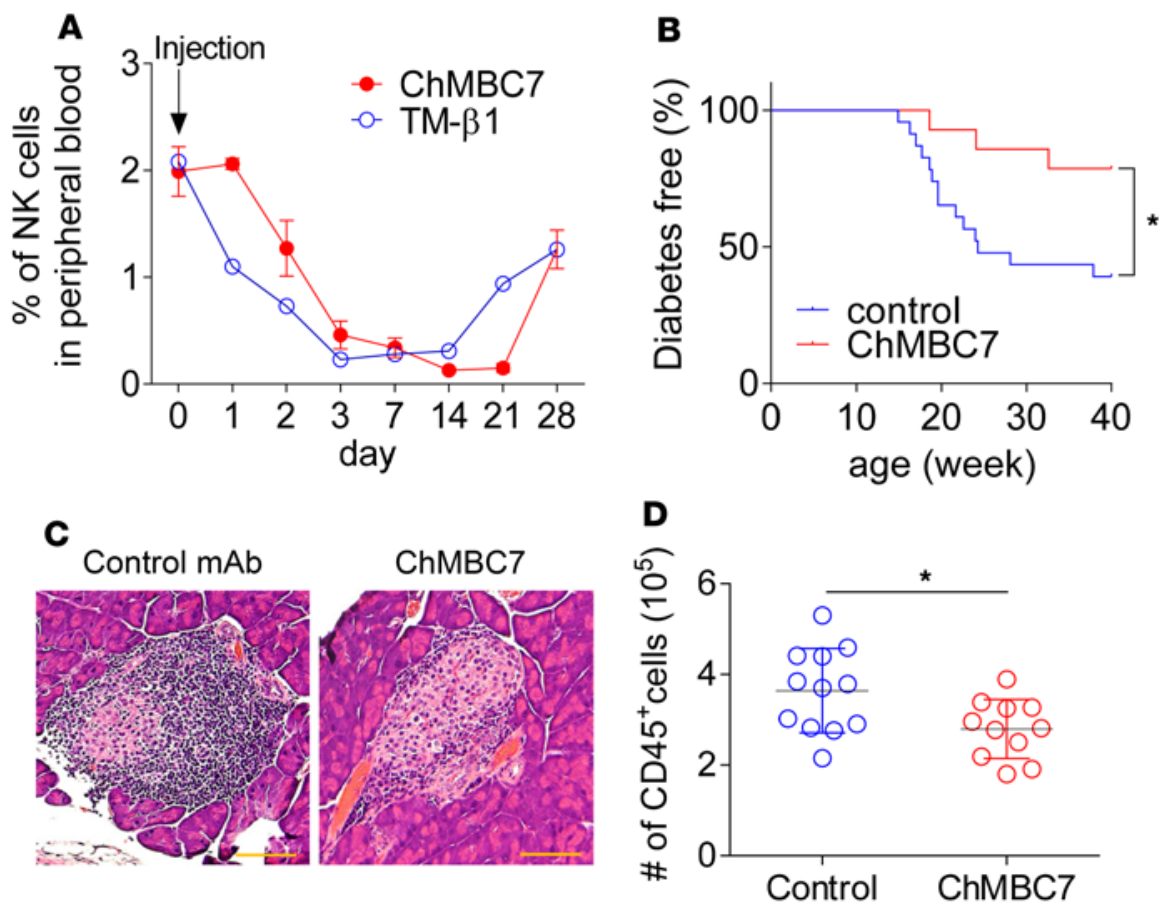

Figure 1. Anti-CD122 treatment suppresses insulitis and diabetes in NOD mice. (A) The percentages of NK cells from peripheral blood of 10-week-old NOD mice injected with 1 dose TM- $\beta 1(5 \mathrm{mg} / \mathrm{kg})$ or ChMBC7 $(5 \mathrm{mg} / \mathrm{kg})(n=3)$ and analyzed at various time points as indicated. (B) The incidence of diabetes onset in female NOD mice treated with antiCD122 (ChMBC7) ( $n=14)$ or control mAb ( $n=23)$ for 7 weeks (from 3-10 weeks of age). (C) Histology of formalin-fixed and H\&E-stained pancreas sections from mice treated as in $\mathbf{B}(n=3$ in each group). Scale bar: $50 \mu \mathrm{m}$. (D) The numbers of CD45+ immune cells from each individual pancreas of control $(n=12)$ or ChMBC7-treated $(n=11)$ mice. Data are shown as mean \pm SEM. Statistical data were calculated using Gehan-Breslow-Wilcoxon test (B) or Student's $t$ test (D). ${ }^{*} P<0.05$.

T cell subsets isolated from pancreatic islets. CD $44^{+}$effector/memory phenotype (mp) T cells (both CD4 ${ }^{+}$ and $\mathrm{CD}^{+}$) exhibited a relatively higher level of CD122 expression than $\mathrm{CD}^{-} 4^{-}$native subset (Figure $2 \mathrm{C}$ ). In summary, these data revealed a detailed profile of CD122 expression in lymphoid organs and T1D-associated pathological lesions. It thus can be speculated that modulating CD122 signaling would affect the activities of these $\mathrm{CD} 122^{+}$cells.

Anti-CD122 differentially regulates IL-2 and IL-15 signaling in different immune cells. Both IL-2 and IL-15 are trophic cytokines for the proliferation and survival of $\mathrm{T}$ and NK cells (reviewed in ref. 11). STAT5 is a key IL-2/IL-15R $\beta$ downstream transcription factor $(12,20)$. We asked whether CD122 blockade affected downstream signaling in T cells and NK cells in response to IL-2 or IL-15 stimulation. In this regard, total splenocytes from 10-week-old prediabetic NOD mice were cultured in the presence of IL-2 or IL-15, with the supplementation of either control $\mathrm{mAb}$ or ChMBC7. The phosphorylation of STAT5 was measured at various time points (Figure 3A). As expected, IL-15 elicited a robust phosphorylation of STAT5 in both $\mathrm{NK}$ and $\mathrm{CD} 8^{+} \mathrm{T}$ cells, but not in $\mathrm{CD}^{+}$conventional $\mathrm{T}$ (Tconv) cells. This effect was potently suppressed by ChMBC7 (Figure 3B). NK cells also responded to IL-2, as previously implicated (21-23), and exhibited a detectable level of STAT5 activation, though to a less magnitude compared with IL-15. Of note, ChMBC7 almost completely abolished IL-2-induced pSTAT5 in NK cells (Figure 3B). IL-2 failed to induce phosphorylation of STAT5 in $\mathrm{CD}^{+}$or $\mathrm{CD}^{+}$Tconv cells. Therefore, CD122 blockade effectively suppresses STAT5 activation induced by IL-15 in both NK and CD8 ${ }^{+} \mathrm{T}$ cells, and by IL-2 in NK cells. In contrast, Tregs exhibited distinct responses to ChMBC7-mediated interference of IL-2 or IL-15 stimulation. Both IL-2 and IL-15 induced robust phosphorylation of STAT5 in Tregs (Figure 3C). Of note, ChMBC7 suppressed IL-15-elicited, but not IL-2-elicited, STAT5 phosphorylation in Tregs. Together, in vivo CD122 blockade differentially regulates the activation of IL-2/IL-15R $\beta$ downstream signaling in different immune cells. Thus, a beneficial outcome of CD122 blockade under autoimmune conditions can be expected, since the responsiveness of Tregs, not pathogenic NK or $\mathrm{CD}^{+} \mathrm{T}$ cells, to IL-2 is preserved. 
A

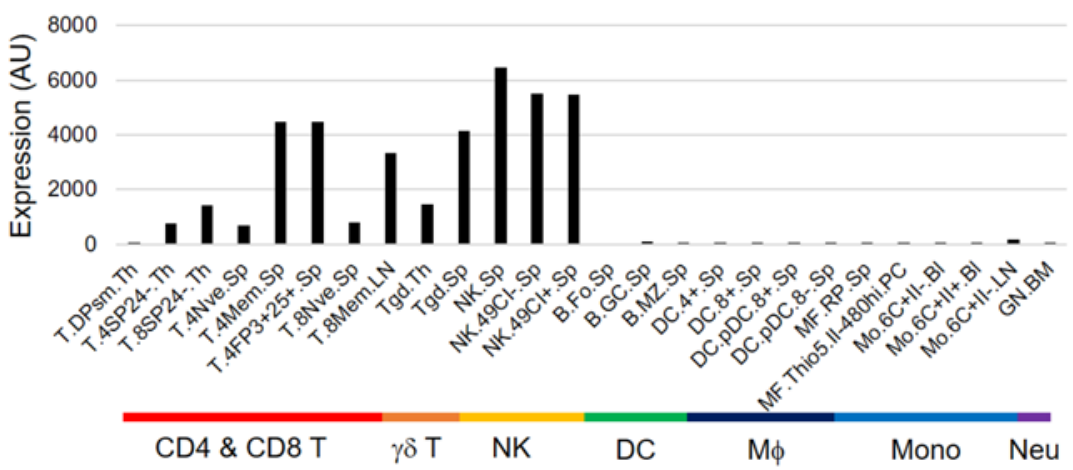

B

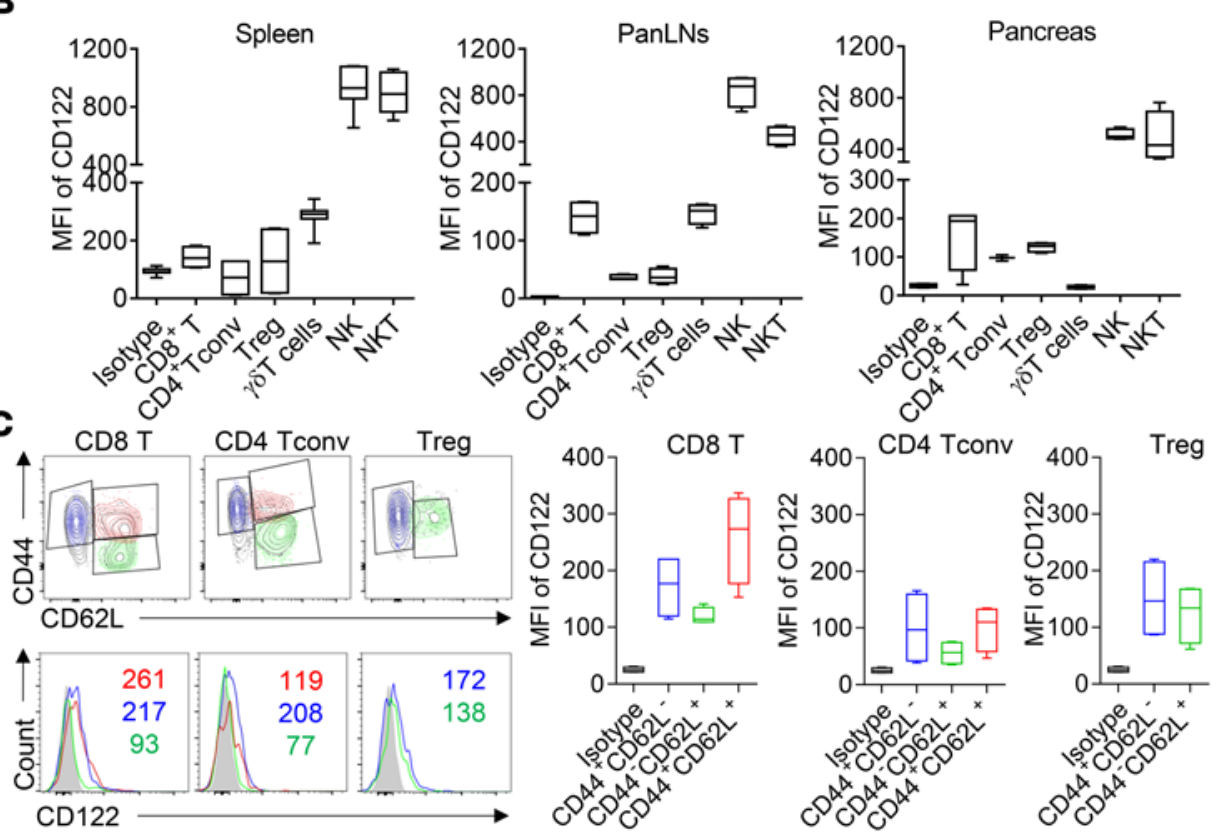

Figure 2. CD122 expression in various immune cells. (A) The expression profile of $C d 122$ in representative immune cell populations from the ImmGen (www.immgen.org). AU, arbitrary unit of normalized expression; $M \phi$, macrophage; Mono, monocyte; Neu, neutrophil; Sp, spleen; Th, thymus; BI, blood; LN, lymph node. (B) The expression of CD122 protein in indicated cell types from spleen, pancreatic lymph node (panLN), and pancreatic islets of 4-week-old NOD mice $(n=4)$. MFI, mean fluorescence intensity. (C) The expression of CD122 in the subsets of CD8 ${ }^{+}$T cell, CD4 ${ }^{+}$Tconv, and Tregs from pancreatic islets. Numbers in each panel are MFI of CD122. Data are representative of 3 independent experiments (B and $\mathbf{C})$.

CD122 blockade preferentially ablates pathogenic $N K$ and effector/memory $T$ cells, leaving Tregs mildly affected. We examined whether CD122 blockade regulated the abundance of NK and T cells. First, we tested the effect of $\mathrm{ChMBC7}$ on $\mathrm{T}$ cell and NK cell survival and expansion in vitro. In this regard, total splenocytes from 10-week-old prediabetic NOD mice were prepared and cultured with either medium alone or with the supplementation of IL-2 or IL-15. IL-15 induced a robust proliferation of CD8 $8^{+}$, NK, and NKT cells, as well as survival of $\mathrm{CD} 4^{+}$Tconv cells (Supplemental Figure 4 and data not shown). These effects of IL-15 were almost completely abolished by either ChMBC7 or TM- $\beta 1$ (Supplemental Figure 4). IL-2 exhibited a similar effect on the proliferation of $\mathrm{CD} 8^{+} \mathrm{T}$ cells and the survival of $\mathrm{CD} 4^{+} \mathrm{Tconv}$ cells, which was significantly abolished by ChMBC7 or TM- $\beta 1$. Both IL-2 and IL-15 promoted Treg survival. Interestingly, while ChMBC7 and TM- $\beta 1$ effectively inhibited IL-15-induced Treg survival, IL-2-induced Treg survival was only modestly affected by ChMBC7 or TM- $\beta 1$ (Supplemental Figure 4). Thus, anti-CD122 mAb selectively regulates in vitro survival and expansion of different immune populations.

We then examined the in vivo effect of CD122 blockade under an autoimmune condition. Threeweek-old prediabetic NOD mice were treated with either $\mathrm{ChMBC} 7$ or isotype-matched control $\mathrm{mAb}$ twice a week for 7 weeks. After the treatment, the spleens, panLNs, and pancreatic islets were collected 


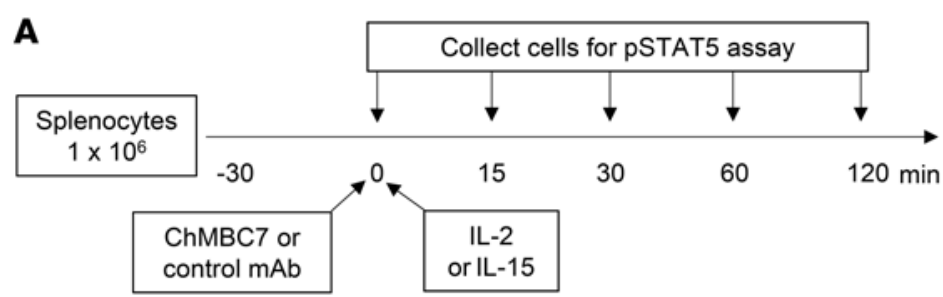

B

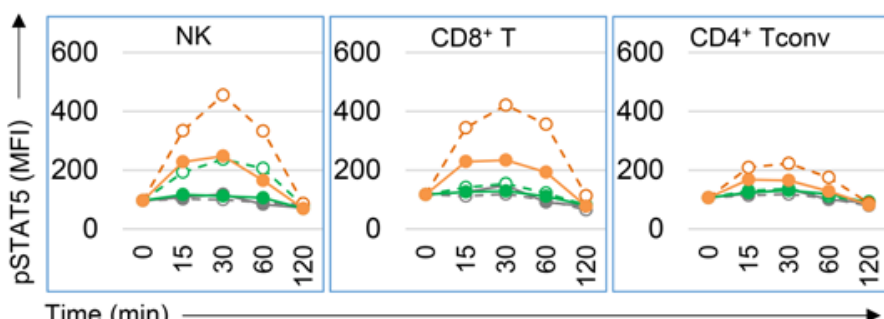

Time (min)

C

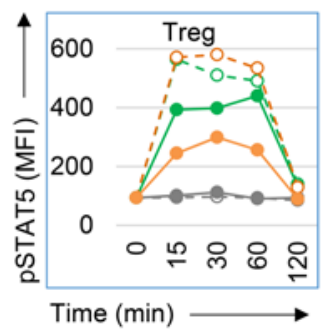

Figure 3. The phosphorylation of STAT5 in NK and T cells.

(A) Experimental design. Splenocytes from 10-week-old prediabetic NOD mice were cultured in RPMI1640 medium for 30 minutes and then stimulated with IL-2 $(100 \mathrm{U} / \mathrm{ml})$ or IL-15 $(50 \mathrm{ng} / \mathrm{ml})$ for indicated length of time in the presence of ChMBC7 or control mAb, respectively. (B and $\mathbf{C}$ ) Phosphorylation of STAT5 under each condition in indicated cell subset at indicated time point. Data are representative of 3 independent experiments.

and analyzed using flow cytometry. In pancreatic islets, in vivo ChMBC7 treatment almost completely ablated both NK and NKT cells (Figure 4, A and B). $\mathrm{CD} 8^{+} \mathrm{T}$ cells were also markedly reduced in ChMBC7-treated mice (Figure 4, A and B). Among CD8 ${ }^{+} \mathrm{T}$ cells, the CD $44^{+} \mathrm{CD} 62 \mathrm{~L}^{-}$effector memory subset was preferentially affected by $\mathrm{ChMBC7}$, resulting in an increased proportion of $\mathrm{CD} 44^{-} \mathrm{CD} 62 \mathrm{~L}^{+}$ naive $\mathrm{CD} 8^{+} \mathrm{T}$ cells (Figure $4 \mathrm{C}$ ). As expected, $\mathrm{CD} 44^{+} \mathrm{CD} 122^{\text {hi }}$ cells were preferentially ablated by $\mathrm{ChM}-$ BC7 (Figure 4D). Though the total number of islet CD $4^{+} \mathrm{Foxp}^{-}$Tconv cells was only mildly affected (Figure $4, \mathrm{~A}$ and $\mathrm{B})$, the fraction of effector/memory subsets $\left(\mathrm{CD} 44^{+} \mathrm{CD} 62 \mathrm{~L}^{-}\right)$was significantly lower in ChMBC7-treated mice, compared with control mice (Supplemental Figure 5), suggesting that CD122 blockade dampened the activation and function of CD4 ${ }^{+}$Tconv cells. In contrast, the percentages, total numbers, and subsets of Tregs in pancreatic islets were comparable between ChMBC7 and control $\mathrm{mAb}$-treated mice, suggesting that $\mathrm{ChMBC} 7$ did not alter the abundance and activation of Tregs (Figure 4E and Supplemental Figure 5). Moreover, the suppressive function of Tregs was not affected by ChMBC7 (Supplemental Figure 6). Thus, in vivo CD122 blockade reconstructs the immune compartment in pancreatic islets by selectively ablating NK and effector/memory $\mathrm{T}$ cells and preserving Treg abundance and function.

As a consequence of the selective effects of $\mathrm{ChMBC7}$ on different immune cell populations, the ratios between Tregs and pathogenic cells (NK cells and $\mathrm{CD}^{+} \mathrm{T}$ cells) in pancreatic islets were significantly elevated (Figure $4 \mathrm{~F}$ ), indicating a reset balance favoring immune tolerance over autoimmunity. We also examined the effects of CD122 blockade on immune cells from lymphoid organs, including spleen and pancreatic lymph nodes (pLNs). At these locations, ChMBC7 exerted rather mild effects on T cells, though NK cells were more profoundly affected (Supplemental Figure 7). Of note, the fractions of Tregs in the spleen were even slightly decreased (Supplemental Figure 7), suggesting that CD122 blockade did not provoke a systemic immune suppression.

CD122 blockade suppresses IFN- $\gamma$ production. IFN- $\gamma$ is a major cytokine in T1D pathogenesis by polarizing a variety of immune cells (including $\mathrm{T}$ cells and macrophages) and amplifying T1D-associated inflammatory processes $(24,25)$. IL-2 signaling is crucial for Th1 differentiation, characterized by IFN- $\gamma$ 

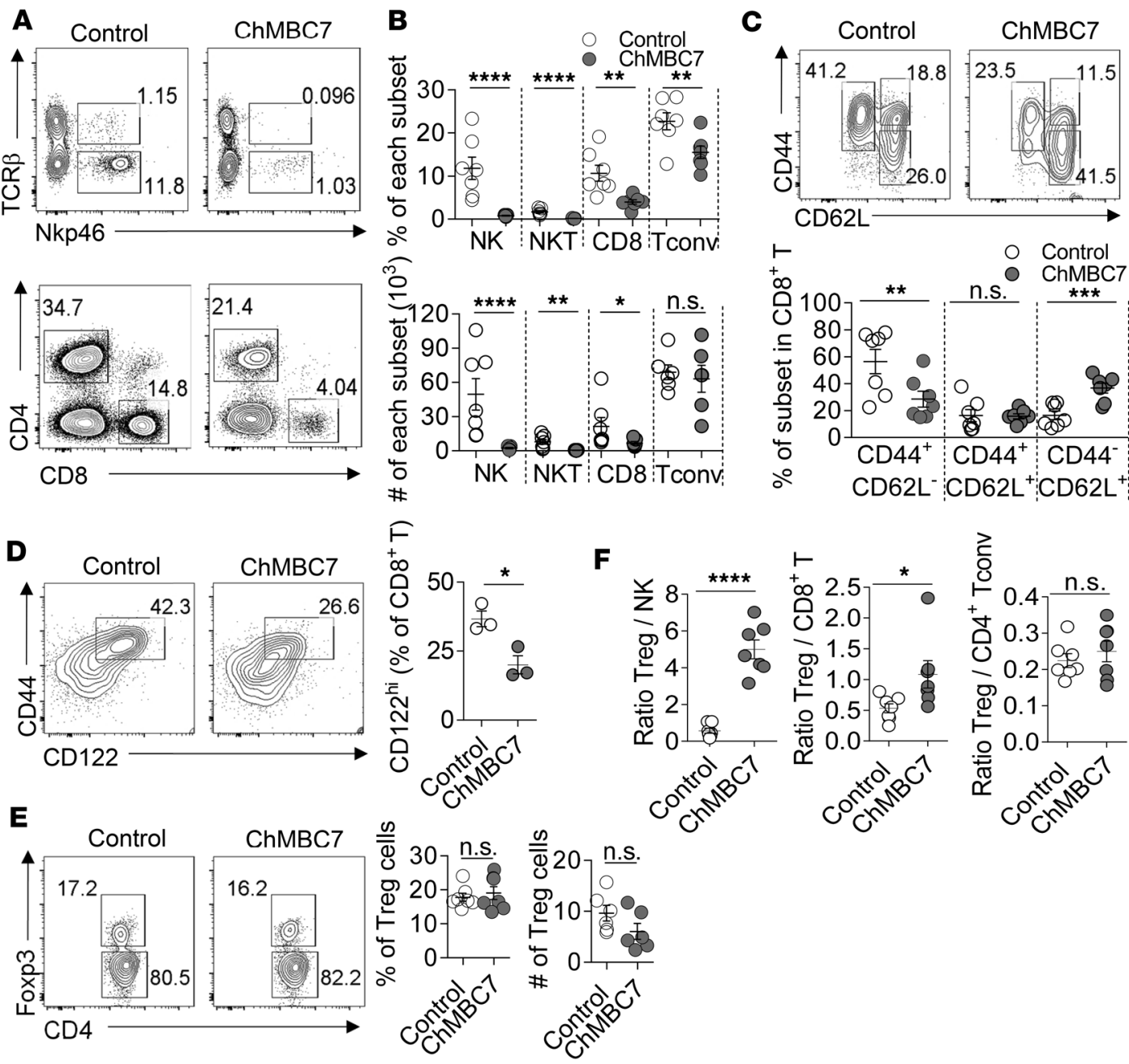

Figure 4. Anti-CD122 treatment alters immune compartment in pancreatic islets. (A) Representative FACS plots of immune cell profiles in pancreatic islets of NOD mice treated with control mAb or ChMBC7 for 7 weeks (from 3-10 weeks of age). (B) Statistical data of the percentages and numbers of indicated subsets from pancreatic islets of the mice treated in $\mathbf{A}(n=$ 7 in each group). (C) Representative FACS plots (upper) and statistics (lower) of CD8 subsets from mice as in $\mathbf{A}(n=7)$. (D) Eight-week-old NOD/BDC2.5 mice were treated with control mAb or ChMBC7 for 2 weeks. Representative FACS plots (left) and statistics (right) of $C D 44^{+} C D 122^{\text {hi }}$ subset within pancreatic CD8 ${ }^{+}$T cells ( $n=3$ in each group). (E) Representative FACS plots (left) and statistics (right) of Tregs from pancreatic islets of mice as in $\mathbf{A}$. (F) The ratios between Tregs and pathogenic cells as indicated from mice as in $\mathbf{A}$. Statistical data are mean \pm SEM. Data are representative of 3 independent experiments. $P$ values are calculated using Student's $t$ test. ${ }^{*} P<0.05 ;{ }^{* *} P<0.01 ;{ }^{* *} P<0.001 ;{ }^{* * *} P<0.0001$.

production $(26,27)$. Therefore, we examined whether modulating IL-2R signaling affected IFN- $\gamma$ production. In ChMBC7-treated NOD mice, the percentages of IFN- $\gamma^{+}$cells were significantly reduced in $\mathrm{CD}^{+}$and $\mathrm{CD} 8^{+} \mathrm{T}$ cells and TCR $\alpha \beta^{-}$cells (Figure 5, A and B). Given the markedly diminished cellularities of pathogenic $\mathrm{NK}$ and $\mathrm{CD} 8^{+} \mathrm{T}$ cells after $\mathrm{ChMBC} 7$ treatment (Figure $4 \mathrm{~B}$ ), the total number of IFN- $\gamma$-producing cells within pancreatic islets was even more profoundly reduced (Figure 5C). However, the expression of T-bet, a master transcription factor for Ifng expression, was not affected by ChMBC7 (Figure 5D), suggesting that posttranscriptional regulations were involved in the suppression of IFN- $\gamma$ production by ChMBC7. Thus, in vivo CD122 blockade suppresses IFN- $\gamma$ production, ameliorating IFN- $\gamma$-mediated amplification of islet inflammatory cascades.

CD122 blockade suppresses the conversion of Th17 cells into diabetogenic Th1 cells. In contrast to a significant reduction of IFN- $\gamma^{+}$Th1 cells in the islets of ChMBC7-treated mice, both the proportions and numbers of IL-17 $\mathrm{A}^{+} \mathrm{CD} 4^{+} \mathrm{T}$ cells (Th17 cells) in pancreatic islets were significantly increased after ChMBC7 treatment (Figure 6A). The role of Th17 cells in T1D remains controversial (3). A conversion of Th17 cells into Th1 cells within pancreatic islets has been demonstrated to induce diabetes, supporting 


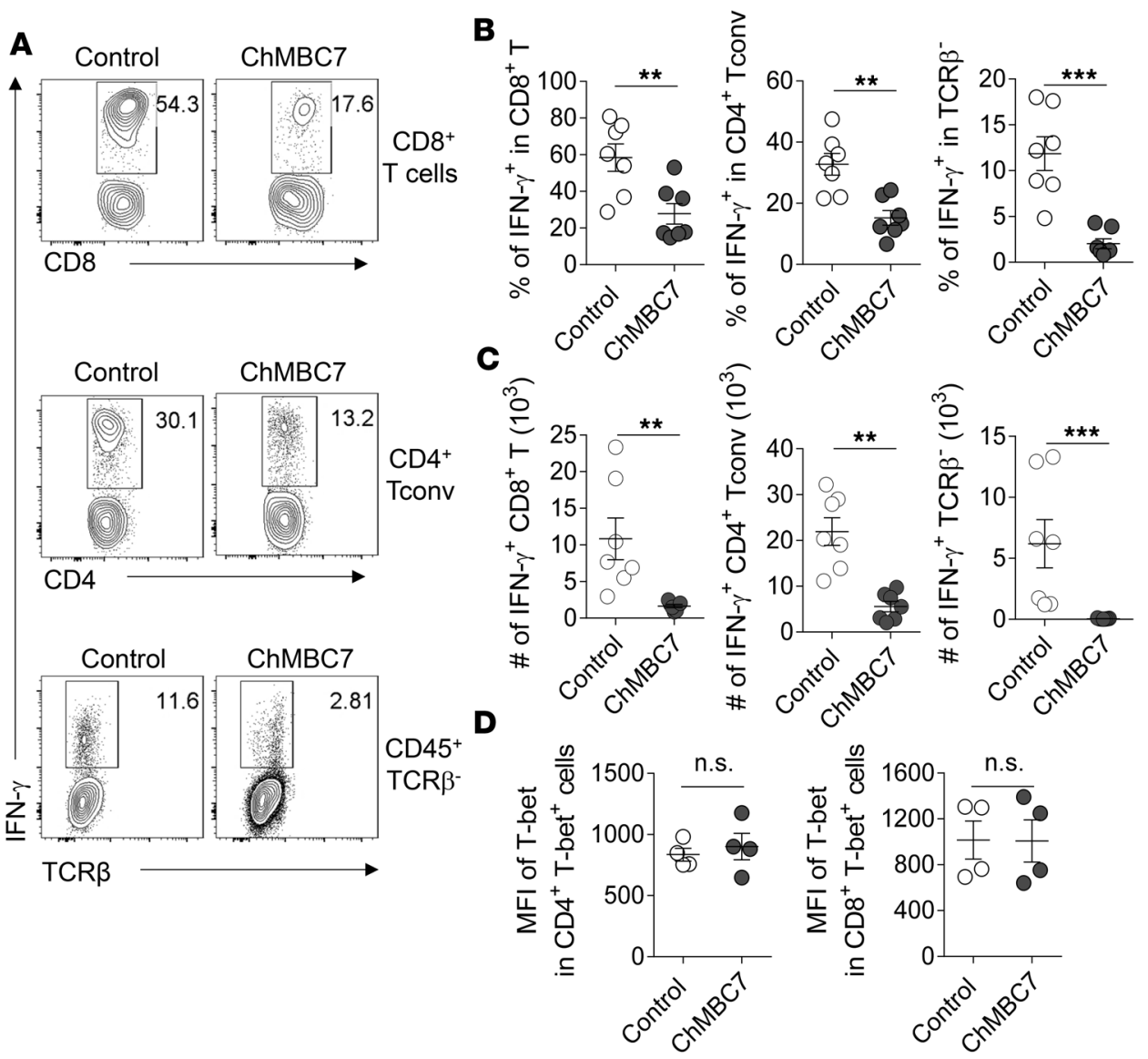

Figure 5. Anti-CD122 treatment suppresses IFN- $\gamma$ production. (A) Representative FACS plots of IFN- $\gamma$ production in indicated cell subsets isolated from pancreatic islets of NOD mice treated with control mAb or ChMBC7 from 3-10 weeks of age ( $n=7$ in each group). (B and $\mathbf{C}$ ) The percentages (B) and total numbers (C) of IFN- $\gamma^{+}$cells in indicated cell subsets prepared as in A. (D) The expression of T-bet in in vitro activated $C D 4^{+}$and $C D 8^{+} T$ cells (by anti-CD3/CD28 and IL-2) with or without ChMBC7. Data are representative of $3(\mathbf{A}-\mathbf{C})$ or $2(\mathbf{D})$ independent experiments. Statistical data are mean \pm SEM. $P$ values are calculated using Student's $t$ test. ${ }^{* *} P<0.01 ;{ }^{* *} P<0.001$.

a notion that Th17 cells themselves are not diabetogenic $(28,29)$. We asked whether CD122 blockade affected the Th17-to-Th1 conversion. Our in vitro $\mathrm{CD} 4^{+} \mathrm{T}$ cell differentiation assays showed that ChMBC7 promoted the generation of Th17 cells, at the expense of reduced Th1 cell generation (Supplemental Figure 8), suggesting that blocking IL-2/IL-15R $\beta$ signaling redirects Th1-Th17 differentiation. We next examined the in vivo effect of $\mathrm{ChMBC7}$ under autoimmune conditions (Figure 6B). In this regard, we used a surface-capturing technique (30) to sort highly purified Th17 cells generated in vitro (Figure 6C) and transferred them into 3-week-old NOD mice. These mice were treated with either ChMBC7 or control $\mathrm{mAb}$ (Figure 6B). After 1 week, transferred Th17 cells were collected from pancreatic islets and spleens and were analyzed for their production of IL-17A and IFN- $\gamma$. Consistent with previous reports $(28,29)$, in control mice, a substantial proportion of transferred Th17 cells were converted to IFN- $\gamma^{+}$ Th1 cells (Figure 6, C and D). This conversion was significantly suppressed by ChMBC7 (Figure 6, C and D). Notably, the conversion of Th17 to Th1 preferentially occurred at tissue sites (pancreatic islets), not in lymphoid organs (Figure 6E), suggesting that a local inflammatory microenvironment provoked the conversion. Because diabetes development was suppressed by ChMBC7 treatment (Figure 1B), these data suggest that Th17 cells are not diabetogenic and an islet inflammatory environment provokes the conversion of Th17 to Th1 cells, which are bona fide pathogenic cells in T1D.

A combination of CD122 blockade and Treg-trophic cytokines promotes tissue Treg abundancy and function. The total number of Tregs was slightly (though not significantly) reduced in ChMBC7-treated mice (Figure $4 \mathrm{E})$. We asked whether Treg-tropic cytokines would reinforce the abundance and/or function of Tregs in ChMBC7-treated mice. Low-dose IL-2 $(31-33)$ and IL-33 $(34,35)$ have been reported to promote Treg 
A

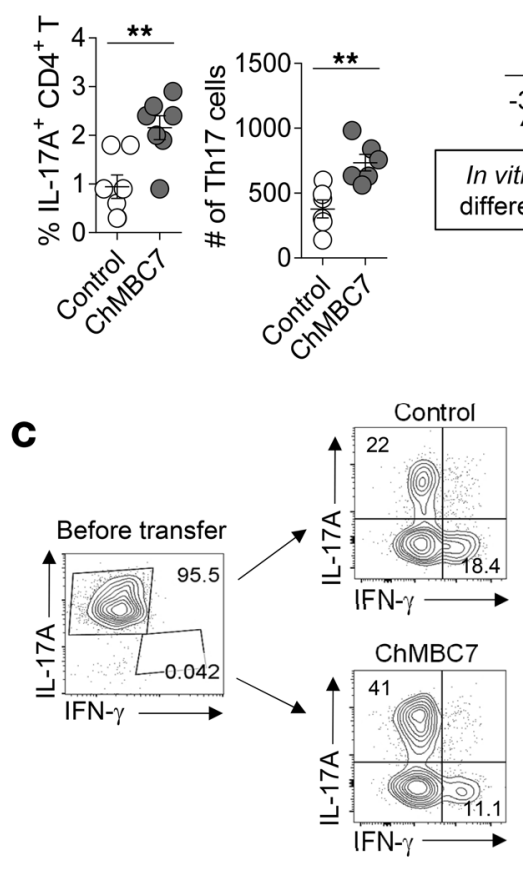

B

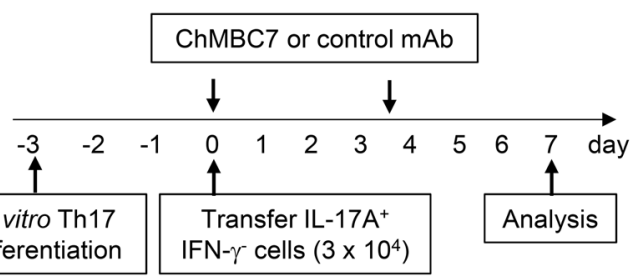

D

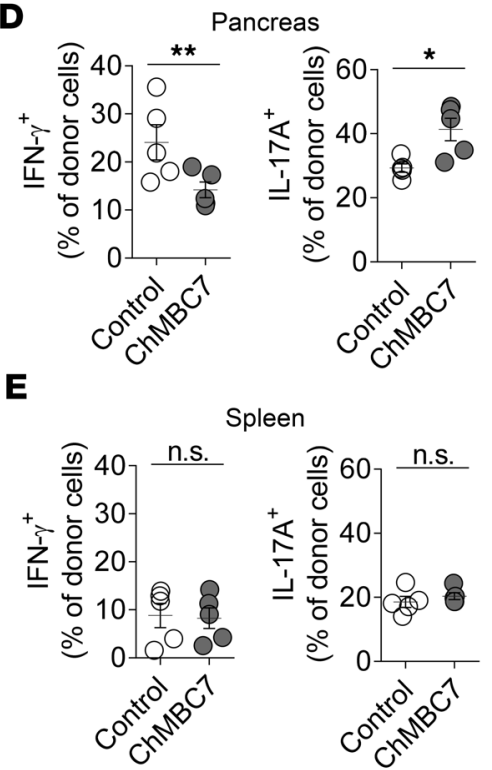

Figure 6. Anti-CD122 treatment prevents the conversion of Th17 cells to pathogenic Th1 cells in the pancreas. (A) The percentages of Th17 cells in pancreatic islets of 10-week-old NOD mice treated with control mAb or ChMBC7 for 7 weeks ( $n=7$ in each group). (B) Schematic diagram showing experimental design of Th17 transfer experiments. (C) Representative FACS plots of IL-17A and IFN- $\gamma$ expression in donor cells before and after the transfer. (D and E) IFN- $\gamma$ and IL-17A production by donor $C D 4^{+} T$ cells from pancreatic islets $(\mathbf{D})$ and spleen $(E)$ in recipient mice treated with either control mAb or ChMBC7 ( $n=5$ in each group). Data are representative of $3(\mathbf{A})$ or 2 (B-E) independent experiments. Statistical data are mean \pm SEM. $P$ values are calculated using Student's $t$ test. ${ }^{*} P<0.05 ;{ }^{* *} P<0.01$.

expansion in vivo. We examined whether a combination of ChMBC7 with low-dose IL-2 or short-term IL-33 would affect Tregs. In this regard, 10-week-old prediabetic NOD mice were treated with control mAb or anti-CD122, in combination with IL-2 or IL-33, respectively (Figure 7A). We found that a short-term supplementation of low-dose IL-2 (daily for 5 days) or IL-33 (daily for 3 days) to ChMBC7 administration significantly augmented the percentages of Tregs within pancreatic islets (Figure 7B). The total number of Tregs was also increased under such combinational treatment protocols, though variations among individual mice were noticed in mice that received ChMBC7 plus IL-33 (Figure 7B).

The expression of ICOS and KLRG1 are critical for the function of Tregs $(36,37)$. We examined whether the addition of IL-2 or IL-33 affected the functional profile of Tregs. Though both IL-2 and IL-33 were capable of increasing Treg cell abundancy in vivo in ChMBC7-treated NOD mice, the upregulation of ICOS and KLRG1 was more profoundly promoted by IL-33 (Figure 7C). Over 70\% of Tregs from pancreatic islets of $\mathrm{ChMBC} 7$ plus IL-33-treated mice were $\mathrm{ICOS}^{+}$. Moreover, in these mice, a significantly higher fraction of Tregs were double-positive for both ICOS and KLRG1 (Figure 7C). Notably, although IL-33 alone was sufficient to promote the expression of ICOS in Tregs, the upregulation of KLRG1 was only observed under the condition of a combinational treatment of ChMBC7 and IL-33. Enhanced suppressive function has been reported for KLRG1 ${ }^{+}$or ICOS ${ }^{+}$Tregs (37-39). In line with this, we found that Tregs from IL-33-treated mice were more suppressive in vitro (Supplemental Figure 6). In summary, these data support therapeutic benefits of a combinational treatment with CD122 blockade and Treg-trophic cytokines (in particular IL-33) to restore immune tolerance in T1D. 
A

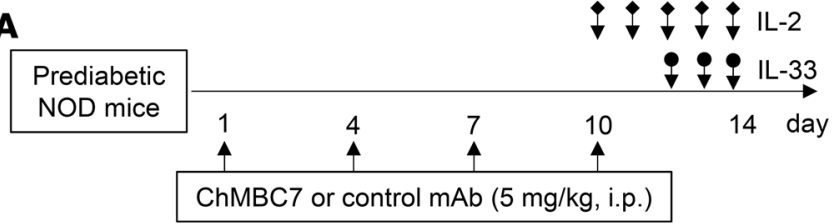

B

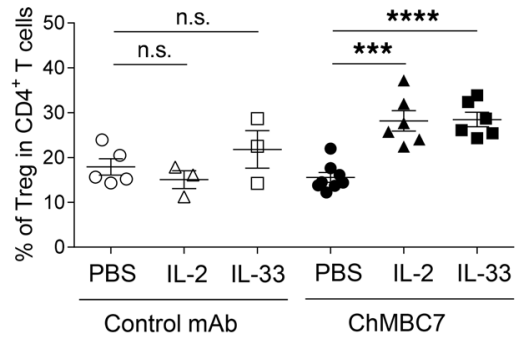

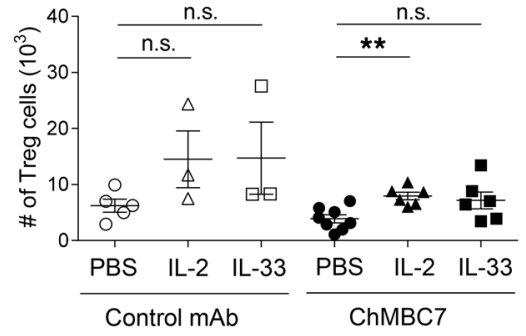
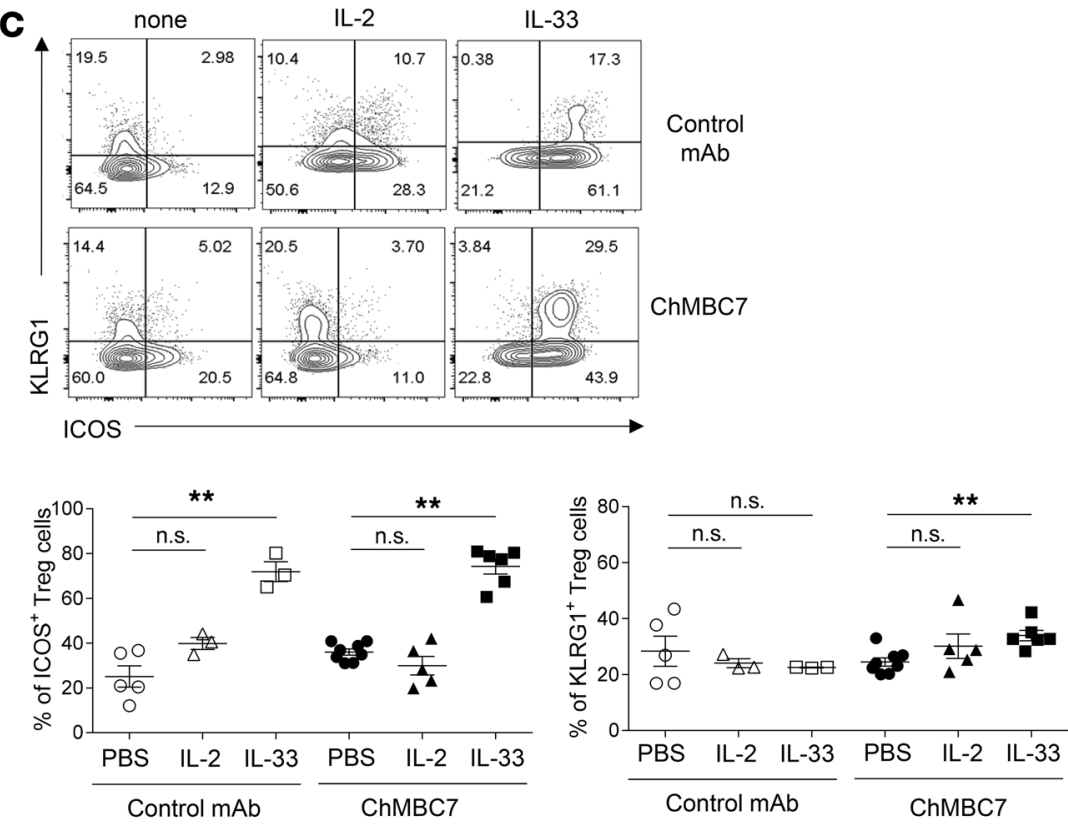

Figure 7. A combination of CD122 blockade and Treg-trophic cytokines promotes effector Treg expansion in vivo. (A) Schematic diagram showing experimental design. Ten-week-old female prediabetic NOD mice were treated with control mAb $(n=$ 5), control mAb + IL-2 $(n=3)$, control mAb + IL-33 ( $n$ $=3)$, anti-CD122 (chMBC7) $(n=8)$, anti-CD122 + IL-2 $(n=6)$, or anti-CD122 + IL-33 $(n=6)$ for 2 weeks. (B) The percentages and total numbers of Tregs from the pancreatic islets of the mice under each treatment condition. (C) Representative FACS plots (upper) and statistics (lower) of KLRG1 and ICOS expression in Tregs from indicated groups as in A. Statistical data are mean \pm SEM. Data are representative of 3 independent experiments. $P$ values are calculated using 1-way ANOVA with a Tukey multiple comparison test. ${ }^{*} P<$ $0.01 ;{ }^{* * *} P<0.0001$.

\section{Discussion}

Previous studies have reported that blocking IL-2/ IL-15R $\beta$ signaling with a mAb (clone TM- $\beta 1$ ) effectively suppressed diabetes development in NOD mice (13-16). However, the mechanisms remain poorly defined. In this study, we have focused on elucidating how CD122 blockade suppressed diabetes development. To eliminate the FcR bindingmediated effects, we have developed an Fc-silent $\mathrm{mAb}$ for CD122 (clone ChMBC7), which binds to the same epitope of CD122 as TM- $\beta 1$. ChMBC7 and TM- $\beta 1$ exhibit a comparable effect in blocking IL-15 transpresentation. Using ChMBC7 to modulate IL-2/IL-15R $\beta$ signaling in vitro and in vivo, we have demonstrated that several mechanisms were involved in CD122 blockade-mediated diabetes suppression. On a cellular level, CD122 blockade preferentially ablated NK and effector/memory T cells in pancreatic islets. In contrast, the abundance and function of islet Tregs were negligibly affected.

On a molecular level, CD122 blockade inhibited

IFN- $\gamma$ production in various T1D-pathogenic cells. We also found that in vivo CD122 blockade altered the fate of the Th17 cell in the islets of NOD mice. Together, these findings provide important mechanistic insights into this new immunomodulatory strategy to restore immune tolerance in T1D.

How might CD122 blockade preferentially ablate pathogenic cells over Tregs? This could be explained by different binding affinities of IL-2 and IL-15 to their cognate receptors. CD122 and CD132 (common $\gamma$ chain, $\gamma c$ ) form a receptor complex that exhibits an intermediate binding affinity to IL-2 or IL-15 ( $\mathrm{K}_{\mathrm{D}}$ "1 $\mathrm{nM})(12,40,41)$. The binding affinity between IL-2 and isolated CD25 (IL-2R $\alpha)$ is also low $\left(\mathrm{K}_{\mathrm{D}}\right.$ "10 nM) $(12,42)$. However, in the presence of all $3 \mathrm{IL}-2 \mathrm{R}$ subunits (CD25/CD122/CD132), the binding affinity between IL-2 and IL-2R complex (composed of $\alpha / \beta / \gamma$ subunits) is markedly increased (K $\mathrm{D}_{\mathrm{D}}$ $\left.10 \mathrm{pM}\right)(12$, $40,41,43)$. In contrast, the transpresentation interaction of IL-15/IL-15R $\alpha$ to CD122/CD132-bearing cells has an affinity around nanomolar magnitude (12). Thus, an anti-CD122 mAb (such as ChMBC7), with binding affinity for CD122 in the nanomolar range, can potently interrupt the binding of IL-2 or IL-15/ IL-15R $\alpha$ to their dimeric receptor complex (CD122/CD132) but fails to compete with IL-2 for binding to its high-affinity trimeric receptor complex (CD25/CD122/CD132). CD25 is abundantly expressed in Tregs (and to some extent in activated $\mathrm{T}$ cells), absent in $\operatorname{mpCD} 8^{+} \mathrm{T}$ cells, and absent or low in NK cells. The cell type-specific expression of CD25 provides an explanation of why CD122 blockade preferentially affects NK cells and $\mathrm{mpCD} 8^{+} \mathrm{T}$ cells, leaving Tregs only mildly affected. 
This study also provides potentially novel insights into the regulation of Th cell differentiation and their roles in T1D. IL-2 signaling is crucial for Th1 differentiation $(26,27)$. It thus can be speculated that the effect of ChMBC7 on the suppression of Th1 cell differentiation was due to a direct blockade of IL-2 signaling in $\mathrm{CD}^{+}$Tconv cells. In contrast, IL-2 signaling plays a dampening role in Th17 differentiation (44-46). We thus propose a derepression model whereby ChMBC7 promotes Th17 cell differentiation by inhibiting the suppressive effect of IL-2. A number of studies have proposed that Th17 cells played a disease-promoting role in T1D $(47,48)$, mainly through their signature cytokine IL-17 (49-54) or, alternatively, through TNF- $\alpha$ produced by Th17 cells (55). However, this opinion was contradicted by other studies suggesting that Th17 cells may possess a disease-suppressive role in T1D (56-59). A third opinion argued that Th17 cells were indispensable for T1D development (60-62). These contradictory views of Th17's role in T1D may be accounted for by the heterogeneities of Th17 cells $(63,64)$, and/or the different stages of the disease. Nevertheless, one interesting model of Th17's role in T1D was that these cells converted to diabetogenic IFN- $\gamma^{+}$Th1 cells in the proinflammatory islet microenvironment $(28,29)$. We confirmed this in vivo Th17-to-Th1 conversion by showing that, after transferring highly purified Th17 cells into young NOD mice, a substantial fraction of IL- $17 \mathrm{~A}^{+}$donor cells in pancreatic islets differentiated into IFN- $\gamma^{+} \mathrm{Th} 1$ cells, supporting the notion that an insulitic islet environment favored a Th1 differentiation. We found that blocking CD122 signaling suppressed the Th17-to-Th1 conversion in vivo. In line with this, the abundance of endogenous Th17 cells in pancreatic islets was increased in ChMBC7-treated NOD mice. Since diabetes development was suppressed by ChMBC7 treatment, it suggested that Th17 cells in pancreatic islets by themselves were not diabetogenic.

Combinatorial immunotherapies have been implicated to have greater efficacies in achieving immune tolerance and/or restoring $\beta$ cell function in T1D (65-69). We evaluated the efficacies of a combination of CD122 blockade and IL-2 or IL-33 in affecting the abundance and function of Tregs. Our data reveal 2 new perspectives. First, a combination of anti-CD122 plus Treg-trophic cytokines elicited a more profound effect on Tregs than that by cytokines alone. These data provide a strong rationale for further optimizing the combinational regimens in T1D prevention and treatment. Second, even though both IL-2 and IL-33 were able to augment the abundancy of Tregs in vivo in anti-CD122-treated mice, only IL-33 appeared to induce the upregulation of ICOS and KLRG1, two key molecules associated with Treg function. In line with this, Tregs from IL-33-treated mice showed an enhanced suppressive function. Because tissue Tregs express a relatively higher level of IL-33R (also known as ST2) (34, 70-72), it can be speculated that Tregs at tissue sites are more responsive to IL-33 to expand and mature functionally.

Recently, a molecule complex composed of a mutant IL-15 covalently linked to IL-15R $\alpha$ was reported to exhibit an increased affinity for CD122 and an impaired recruitment of CD132 (73). Though via an entirely different design, this modified IL-15/IL-15R $\alpha$ complex inhibited the activation and proliferation of conventional lymphocytes with negligible impact on Tregs (73), an outcome similar to what we have found in this study. Therefore, by modulating IL-2/IL-15R signaling using either engineered antibodies (e.g., ChMBC7) or modified cytokines $(74,75)$, pathogenic immune responses can be abrogated without diminishing Treg function.

In summary, our data provide critical mechanistic insights into CD122 blockade-mediated regulation to restore immune tolerance in T1D. We also demonstrate therapeutic benefits of CD122 blockade plus IL-33 in achieving a simultaneous ablation of pathogenic cells and promotion of Treg abundancy and function.

\section{Methods}

Mice. NOD and NOD/BDC2.5/Thy1.1 mouse lines were imported from Mathis-Benoist laboratory (76). NOD/Foxp $3^{\mathrm{GFP}}$ mice were purchased from the Jackson Laboratory. All mice were housed under specific pathogen free (SPF) conditions in our animal facility at UCSD, in accordance with the ethical guidelines of the IACUC. For the evaluation of diabetes, mice were monitored until 40 weeks of age by measuring urine glucose and blood glucose levels, as described (77). Individuals with 2 consecutive measurements of a serum-glucose concentration above $300 \mathrm{mg} / \mathrm{dl}$ were considered diabetic.

Anti-mouse CD122 mAbs. To obtain rat anti-mouse CD122 mAbs, a Sprague-Dawley female rat was immunized with rat YB2/0 (ATCC CRL-1662) transfectant cells expressing mouse CD122 extracellular domain fused to a glycosylphosphatidylinositol (GPI) anchor. Hybridomas generated by fusing immunized lymphocytes with NS0 cells (ECACC, 8511503) were screened for binding to (i) recombinant mouse CD122-human Fc fusion protein by ELISA and (ii) the mouse CTLL-2 cell line (ATCC, TIB-214) that 
expresses the high-affinity IL-2 receptor (CD25/CD122/CD132) by flow cytometry. mAbs from hybridomas that passed these 2 criteria were further tested for their ability to block human IL-15/human IL-15R $\alpha$ sushi domain complex-mediated (RLI-mediated) (78) proliferation of CTLL-2 cells. Five mAbs were obtained that showed good blocking activity against RLI. All 5 antibodies competed with rat anti-mouse

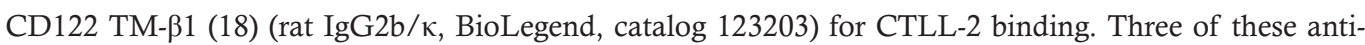
bodies, MBC7, MBC8 and MBC9, not only cross-blocked TM- $\beta 1$ for CTLL-2 binding, but also required a critical arginine residue at position 42 (R42) on mouse CD122 for binding. Crystal structure determination of CD122 indicated that R42 is an important residue that makes contact with IL-2 (79). Substitution of $\mathrm{R} 42$ to alanine in recombinant mouse CD122 invariably abolished its binding by MBC7, MBC8, MBC9, and TM- $\beta 1$. Variable region sequences of MBC7 (rat IgG1/ $/$ ) were determined and used to make a rat/ mouse chimeric antibody called ChMBC7. Chimerization of $\mathrm{MBC} 7$ serves 2 purposes: (i) to reduce the immunogenicity of the resulted antibody in mice to allow prolonged dosing and (ii) to engineer away effector functions of rat antibody in mice to assess the effect of CD122 blockade without the interference of Fc-mediated reactions. TM- $\beta 1$ has a rapid mechanism of depleting NK cells in mice, and it is most likely due to the effector functions of its rat IgG2b isotype (80). ChMBC7 has a mouse IgG2a/ $\mathrm{\kappa}$ isotype but contains 2 amino acid substitutions in the heavy chain (leucine at positions 234 and 235 to alanine; L234A/ L235A) to reduce effector functions. The presence of L234A/L235A in human IgG1 and mouse IgG2a render resulting antibodies to be Fc-silent $(81,82)$. ChMBC7 and TM- $\beta 1$ both bind to CD122 with similar affinity by binding titration flow cytometry on CTLL-2 cells, with a half maximal effective concentration $\left(\mathrm{EC}_{50}\right.$ ) of about $0.2 \mu \mathrm{g} / \mathrm{ml}$. They both blocked RLI-mediated CTLL-2 cell proliferation efficiently with an $\mathrm{IC}_{50}$ of $0.3 \mu \mathrm{g} / \mathrm{ml}$ (Supplemental Figure 2). Like all other anti-CD122 antibodies that effectively block IL-15 transpresentation and interaction of IL-2 with its intermediate affinity receptor (CD122/CD132), neither TM- $\beta 1$ nor ChMBC7 could efficiently inhibit the interaction of IL-2 with its high-affinity receptor complex (CD25/CD122/CD132) (83). Likewise, neither TM- $\beta 1$ nor ChMBC7 could block the IL-2-mediated proliferation of CTLL-2 $\left(\mathrm{IC}_{50}>20 \mu \mathrm{g} / \mathrm{ml}\right)$.

In vivo treatment. For in vivo anti-CD122 treatment, age-matched NOD mice were administered with either control $\mathrm{mAb}$ or $\mathrm{ChMBC} 7(5 \mathrm{mg} / \mathrm{kg}$ ) twice a week for 7 weeks or as indicated. After the treatment, mice were either analyzed or monitored for diabetes occurrence. In some experiments, age-matched NOD mice were administered with 1 dose of $\mathrm{ChMBC} 7$ or control $\mathrm{mAb}$, and peripheral blood was analyzed after the treatment. In other experiments, mice were injected with recombinant cytokine IL-33 (2 $\mu \mathrm{g}$, BioLegend) for the last 3 days or recombinant human IL-2 (25,000 U, PeproTech) daily for the last 5 days during the second week, followed by the ChMBC7 treatment. To assess the in vivo conversion of Th17 into Th1 cells, age-matched mice were adoptively transferred with purified Th17 cells and followed by administration of control $\mathrm{mAb}$ or ChMBC7 twice a week for 1 week. Donor cells from pancreas and spleen were analyzed after the treatment.

Cell preparations. The pancreas was excised and digested in $10 \mathrm{ml}$ phenol red-free DMEM containing collagenase $\mathrm{P}(0.5 \mathrm{mg} / \mathrm{ml})$ (Roche Diagnostics) at $37^{\circ} \mathrm{C}$ on a $200 \mathrm{rpm}$ shaker for 20 minutes. After digestion, single cells were filtered by passing through a $70-\mu \mathrm{m}$ cell strainer and suspended in FACS staining buffer. Single-cell suspensions of lymphoid organs were prepared by mechanic disruption. RBCs were lysated with Ammonium-Chloride-Potassium (ACK) buffer (Lonza).

Antibodies and flow cytometry. All stainings began with an incubation with a mAb for CD16/32 (2.4G2; $\mathrm{BD}$ Biosciences). mAbs to indicated molecules used in this study were CD45 (clone 30-F11), TCR $\beta$ (clone H57-597), TCR $\gamma \delta$ (clone GL3), NKp46 (clone 29A1.4), CD4 (clone GK1.5), CD8 $\alpha$ (clone 53-6.7), Thy1.1 (clone OX-7), Thy1.2 (clone 30-H12), ICOS (clone 7E.17G9), CD25 (clone PC61), CD122 (clone 5H4), CD44 (clone IM7), CD62L (clone MEL-14), KLRG1 (clone 2F1/KLRG1), IL-17A (clone TC11-18H10.1), IFN- $\gamma$ (clone XMG1.2), T-bet (clone 4B10), and PerCP/Cy5.5-streptavidin from BioLegend, as well as Foxp3 (clone FJK-16s) from eBioscience. Intracellular staining of Foxp3 was performed using Foxp3/ transcription factor staining buffer set (eBioscience) according to the manufacturer's instructions. For intracellular IFN- $\gamma$ and IL-17A detection, unfractionated cells were cultured in complete medium in the presence of phorbol myristate acetate (PMA, MilliporeSigma), ionomycin (MilliporeSigma), and brefeldin A (BioLegend) at $37^{\circ} \mathrm{C}$ for 4 hours. Samples were acquired with a BD LSRFortessa (BD Biosciences) and analyzed with FlowJo software.

In vitro cell culture. Total splenocytes $\left(1 \times 10^{6}\right)$ from 10-week-old NOD mice were cultured in RPMI1640 supplemented with 10\% FBS, L-glutamine $(2 \mathrm{mM})$, penicillin $(100 \mathrm{U} / \mathrm{ml})$, streptomycin $(100 \mu \mathrm{g} / \mathrm{ml})$, and 
nonessential amino acids $(100 \mu \mathrm{m})$ and $\beta$-mercaptoethanol $(50 \mu \mathrm{M})$, in the presence of IL-2 $(100 \mathrm{U} / \mathrm{ml}$, PeproTech) or IL-15 (50 ng/ml, PeproTech), with ChMBC7 $(25 \mu \mathrm{g} / \mathrm{ml})$ or control mAb for 7 days. On day 4 , half of the supernatant was removed, and new complete medium, cytokine, and antibody were added back. On day 7, cultured cells were harvested and analyzed. To examine the IL-17 and IFN- $\gamma$ production under Th0 conditions, total splenocytes $\left(5 \times 10^{5}\right)$ from NOD/BDC2.5 mice were cultured with BDC2.5 mimotope $(500 \mathrm{ng} / \mathrm{ml})$ (AnaSpec) in the presence of control mAb or ChMBC7 $(50 \mu \mathrm{g} / \mathrm{ml})$ for 3 days. Cells were then analyzed for the production of IL-17A and IFN- $\gamma$. In some experiments, MACS-enriched (Miltenyi Biotec) $\mathrm{CD}^{+}$and $\mathrm{CD} 8^{+} \mathrm{T}$ cells were cultured in the presence of plate-bound anti-CD3 $(2.5 \mu \mathrm{g} /$ $\mathrm{ml})$, soluble anti-CD28 (2.5 $\mu \mathrm{g} / \mathrm{ml})$, and IL-2 (40 U/ml), with control mAb or ChMBC7 (50 $\mu \mathrm{g} / \mathrm{ml})$ for 3 days. Cells were analyzed for T-bet and IFN- $\gamma$ by flow cytometry.

In vitro Treg suppression assay. CD4 ${ }^{+} \mathrm{GFP}(\text { Foxp3 })^{+}$cells were sorted from the spleen of NOD/Foxp3 ${ }^{\mathrm{GFP}}$ mice that have been treated with control mAb, ChMBC7 (5 mg/kg, twice 1 week for 2 weeks), or IL-33 (2 ng per injection for 3 consecutive days). GFP(Foxp3)+ Tregs were cocultured with sorted CD $4^{+} \mathrm{GFP}(\mathrm{Foxp} 3)^{-}$ Tconv cells labeled with CTV at indicated ratios, in the presence of plate-bound anti-CD3 $(2.5 \mu \mathrm{g} / \mathrm{ml})$, soluble anti-CD28 $(2.5 \mu \mathrm{g} / \mathrm{ml}$, BioLegend), and IL-2 (40 U/ml) for 4 days. The division index of responder $\mathrm{T}$ cells was calculated using the algorithm of averaging number of divisions for all live cells in the culture (84).

Phosphorylation assays. Total splenocytes $\left(1 \times 10^{6}\right)$ first rested in complete RPMI1640 medium for 30 minutes and were then incubated with murine IL-2 $(100 \mathrm{U} / \mathrm{ml})$ or IL-15 $(50 \mathrm{ng} / \mathrm{ml})$ in the presence of ChMBC7 $(25 \mu \mathrm{g} / \mathrm{ml})$ or control $\mathrm{mAb}$ for the indicated time at $37^{\circ} \mathrm{C}$. After that, cells were fixed with paraformaldehyde $(1.6 \%)$ for 10 minutes at $37^{\circ} \mathrm{C}$. Fixed cells were permeabilized with ice-cold methanol for 30 minutes and were stained with anti-phospho-STAT5 (47/Stat5[pY694]; BD Biosciences) and other surface antibodies for 1 hour at room temperature.

In vitro Th17 differentiation and purification. $\mathrm{CD} 4{ }^{+} \mathrm{CD} 25^{-} \mathrm{T}$ cells from NOD/BDC2.5/Thy1.1 mice were MACS enriched and cultured in complete medium with plate-bound anti-CD3 $(2.5 \mu \mathrm{g} / \mathrm{ml})$, soluble antiCD28 (2.5 $\mu \mathrm{g} / \mathrm{ml})$, IL-1 $\beta$ (10 ng/ml, PeproTech), IL-6 (30 ng/ml, PeproTech), TGF- $\beta$ (5 ng/ml, PeproTech), IL-23 (50 ng/ml, PeproTech), anti-IFN- $\gamma$ (XMG1.2, $50 \mu \mathrm{g} / \mathrm{ml}$, BioLegend), and anti-IL-4 (11B11, $10 \mu \mathrm{g} / \mathrm{ml}$, BioLegend) for 3 days. On day 4, cells were harvested, washed, and cultured in complete medium with PMA and ionomycin for 3 hours. An IL-17A secretion assay detection kit and IFN- $\gamma$ secretion assay kit (Miltenyi Biotec) were used to enrich IL-17A-producing cells. Briefly, cells were labeled with IL-17A catch reagent and IFN- $\gamma$ catch reagent, followed by the staining of the anti-IL-17A and anti-IFN- $\gamma$ detection antibodies and surface antibodies according to the manufacturer's instruction. CD4 $4^{+} \mathrm{IL}-17 \mathrm{~A}^{+} \mathrm{IFN}-\gamma$ cells were sorted on a BD Influx sorter, and $3 \times 10^{4}$ cells were transferred into 3-week-old NOD mice.

Histology. For histology assays, the pancreata were removed and fixed with $10 \%$ formalin solution. Fixed tissue blocks were paraffin-embedded, sectioned, and stained with H\&E.

Statistics. Statistical analysis was performed using a 2-tailed unpaired Student's $t$ test when comparing 2 groups and 1-way ANOVA with a Tukey multiple comparison test when comparing multiple groups. Diabetes incidence was analyzed using Gehan-Breslow-Wilcoxon test. Data are presented as mean \pm SEM or otherwise stated in the figure legends. $P<0.05$ was considered significant.

Study approval. All animal experimental and care procedures were approved and conducted in accordance with the ethical guidelines of the IACUC of the UCSD.

\section{Author contributions}

$\mathrm{XY}$ and WF designed experiments. NT and JYT provided reagents and discussed the data. XY and YD performed experiments. All authors analyzed the data. XY and WF wrote the manuscript.

\section{Acknowledgments}

We thank members of the Fu lab at UCSD for experimental assistance, Duy T. Pham and Minami K. Yamamura of JN. Biosciences for antibody engineering of $\mathrm{ChMBC7}$, and the ImmGen consortium for providing gene profiling data used in this study. This work was supported by the AAI Career in Immunology Fellowship (to XY) and NIDDK P30 DK063491, UCSD CTRI UL1 TR000100, and JDRF 2-SRA-2016-306-S-B (to WF).

Address correspondence to: Wenxian Fu, Department of Pediatrics, UC San Diego, 9500 Gilman Drive \#0983, La Jolla, California 92093-0983, USA. Phone: 858.246.0022; Email: w3fu@ucsd.edu. 
1. Eisenbarth GS. Type I diabetes mellitus. A chronic autoimmune disease. N Engl J Med. 1986;314(21):1360-1368.

2. Atkinson MA. The pathogenesis and natural history of type 1 diabetes. Cold Spring Harb Perspect Med. 2012;2(11):a007641

3. Mathis D, Benoist C. The influence of the microbiota on type-1 diabetes: on the threshold of a leap forward in our understanding. Immunol Rev. 2012;245(1):239-249.

4. Lehuen A, Diana J, Zaccone P, Cooke A. Immune cell crosstalk in type 1 diabetes. Nat Rev Immunol. 2010;10(7):501-513.

5. Fu W, Wojtkiewicz G, Weissleder R, Benoist C, Mathis D. Early window of diabetes determinism in NOD mice, dependent on the complement receptor CRIg, identified by noninvasive imaging. Nat Immunol. 2012;13(4):361-368.

6. Bluestone JA, Herold K, Eisenbarth G. Genetics, pathogenesis and clinical interventions in type 1 diabetes. Nature. 2010;464(7293):1293-1300.

7. Smilek DE, Ehlers MR, Nepom GT. Restoring the balance: immunotherapeutic combinations for autoimmune disease. Dis Model Mech. 2014;7(5):503-513.

8. Anderson MS, Bluestone JA. The NOD mouse: a model of immune dysregulation. Annu Rev Immunol. 2005;23:447-485.

9. Alba A, et al. Natural killer cells are required for accelerated type 1 diabetes driven by interferon- $\beta$. Clin Exp Immunol. 2008;151(3): 467-475.

10. Feuerer M, Shen Y, Littman DR, Benoist C, Mathis D. How punctual ablation of regulatory T cells unleashes an autoimmune lesion within the pancreatic islets. Immunity. 2009;31(4):654-664.

11. Boyman O, Krieg C, Homann D, Sprent J. Homeostatic maintenance of T cells and natural killer cells. Cell Mol Life Sci. 2012;69(10):1597-1608.

12. Liao W, Lin JX, Leonard WJ. Interleukin-2 at the crossroads of effector responses, tolerance, and immunotherapy. Immunity. 2013;38(1):13-25.

13. Chen J, et al. Insulin-dependent diabetes induced by pancreatic beta cell expression of IL-15 and IL-15R $\alpha$. Proc Natl Acad Sci U S A. 2013;110(33):13534-13539

14. Arndt B, Witkowski L, Ellwart J, Seissler J. CD8 ${ }^{+}$CD122+ PD-1- effector cells promote the development of diabetes in NOD mice. J Leukoc Biol. 2015;97(1):111-120.

15. Bobbala D, et al. Interleukin-15 plays an essential role in the pathogenesis of autoimmune diabetes in the NOD mouse. Diabetologia. 2012;55(11):3010-3020.

16. Brauner H, Hall HT, Flodström-Tullberg M, Kärre K, Höglund P, Johansson S. Depletion of IL-2 receptor $\beta$-positive cells protects from diabetes in non-obese diabetic mice. Immunol Cell Biol. 2016;94(2):177-184.

17. Herold KC, Vignali DA, Cooke A, Bluestone JA. Type 1 diabetes: translating mechanistic observations into effective clinical outcomes. Nat Rev Immunol. 2013;13(4):243-256.

18. Tanaka T, et al. A novel monoclonal antibody against murine IL-2 receptor beta-chain. Characterization of receptor expression in normal lymphoid cells and EL-4 cells. J Immunol. 1991;147(7):2222-2228.

19. Heng TS, Painter MW, Immunological Genome Project Consortium. The Immunological Genome Project: networks of gene expression in immune cells. Nat Immunol. 2008;9(10):1091-1094.

20. Ring AM, et al. Mechanistic and structural insight into the functional dichotomy between IL-2 and IL-15. Nat Immunol. 2012;13(12):1187-1195.

21. Horowitz A, Behrens RH, Okell L, Fooks AR, Riley EM. NK cells as effectors of acquired immune responses: effector CD4+ T cell-dependent activation of NK cells following vaccination. J Immunol. 2010;185(5):2808-2818.

22. Sitrin J, Ring A, Garcia KC, Benoist C, Mathis D. Regulatory T cells control NK cells in an insulitic lesion by depriving them of IL-2. J Exp Med. 2013;210(6):1153-1165.

23. Gasteiger G, et al. IL-2-dependent tuning of NK cell sensitivity for target cells is controlled by regulatory T cells. J Exp Med. 2013;210(6):1167-1178.

24. von Herrath MG, Oldstone MB. Interferon- $\gamma$ is essential for destruction of beta cells and development of insulin-dependent diabetes mellitus. J Exp Med. 1997;185(3):531-539.

25. Wang B, et al. Interferon-gamma impacts at multiple points during the progression of autoimmune diabetes. Proc Natl Acad Sci U $S$ A. 1997;94(25):13844-13849.

26. Bird JJ, et al. Helper T cell differentiation is controlled by the cell cycle. Immunity. 1998;9(2):229-237.

27. Shi M, Lin TH, Appell KC, Berg LJ. Janus-kinase-3-dependent signals induce chromatin remodeling at the Ifng locus during $\mathrm{T}$ helper 1 cell differentiation. Immunity. 2008;28(6):763-773.

28. Martin-Orozco N, Chung Y, Chang SH, Wang YH, Dong C. Th17 cells promote pancreatic inflammation but only induce diabetes efficiently in lymphopenic hosts after conversion into Th1 cells. Eur J Immunol. 2009;39(1):216-224.

29. Bending D, et al. Highly purified Th17 cells from BDC2.5NOD mice convert into Th1-like cells in NOD/SCID recipient mice. J Clin Invest. 2009;119(3):565-572.

30. Manz R, Assenmacher M, Pflüger E, Miltenyi S, Radbruch A. Analysis and sorting of live cells according to secreted molecules, relocated to a cell-surface affinity matrix. Proc Natl Acad Sci U S A. 1995;92(6):1921-1925.

31. Tang Q, et al. Central role of defective interleukin-2 production in the triggering of islet autoimmune destruction. Immunity 2008;28(5):687-697.

32. Rosenzwajg M, et al. Low-dose interleukin-2 fosters a dose-dependent regulatory T cell tuned milieu in T1D patients. $J$ Autoimmun. 2015;58:48-58.

33. Yu A, et al. Selective IL-2 responsiveness of regulatory T cells through multiple intrinsic mechanisms supports the use of lowdose IL-2 therapy in type 1 diabetes. Diabetes. 2015;64(6):2172-2183.

34. Kuswanto W, et al. Poor repair of skeletal muscle in aging mice reflects a defect in local, interleukin-33-dependent accumulation of regulatory T cells. Immunity. 2016;44(2):355-367.

35. Matta BM, Turnquist HR. Expansion of Regulatory T Cells In Vitro and In Vivo by IL-33. Methods Mol Biol. 2016;1371:29-41.

36. Feuerer M, Hill JA, Kretschmer K, von Boehmer H, Mathis D, Benoist C. Genomic definition of multiple ex vivo regulatory T cell subphenotypes. Proc Natl Acad Sci U S A. 2010;107(13):5919-5924.

37. Cheng G, Yuan X, Tsai MS, Podack ER, Yu A, Malek TR. IL-2 receptor signaling is essential for the development of Klrg1 terminally differentiated T regulatory cells. J Immunol. 2012;189(4):1780-1791. 
38. Kornete M, Sgouroudis E, Piccirillo CA. ICOS-dependent homeostasis and function of Foxp3 $3^{+}$regulatory T cells in islets of nonobese diabetic mice. J Immunol. 2012;188(3):1064-1074.

39. Vocanson M, et al. Inducible costimulator (ICOS) is a marker for highly suppressive antigen-specific T cells sharing features of TH17/TH1 and regulatory T cells. J Allergy Clin Immunol. 2010;126(2):280-289.e7.

40. Wang X, Rickert M, Garcia KC. Structure of the quaternary complex of interleukin-2 with its $\alpha, \beta$, and $\gamma c$ receptors. Science. 2005;310(5751):1159-1163.

41. Boyman O, Sprent J. The role of interleukin-2 during homeostasis and activation of the immune system. Nat Rev Immunol. 2012;12(3):180-190.

42. Ma A, Koka R, Burkett P. Diverse functions of IL-2, IL-15, and IL-7 in lymphoid homeostasis. Annu Rev Immunol. 2006;24:657-679.

43. Waldmann TA. The shared and contrasting roles of IL2 and IL15 in the life and death of normal and neoplastic lymphocytes: implications for cancer therapy. Cancer Immunol Res. 2015;3(3):219-227.

44. Laurence A, et al. Interleukin-2 signaling via STAT5 constrains T helper 17 cell generation. Immunity. 2007;26(3):371-381

45. Yang XP, et al. Opposing regulation of the locus encoding IL-17 through direct, reciprocal actions of STAT3 and STAT5. Nat Immunol. 2011;12(3):247-254.

46. Liao W, Lin JX, Wang L, Li P, Leonard WJ. Modulation of cytokine receptors by IL-2 broadly regulates differentiation into helper T cell lineages. Nat Immunol. 2011;12(6):551-559.

47. Ryba-Stanisławowska M, Skrzypkowska M, Myśliwiec M, Myśliwska J. Loss of the balance between CD4(+)Foxp3(+) regulatory T cells and CD4(+)IL17A(+) Th17 cells in patients with type 1 diabetes. Hum Immunol. 2013;74(6):701-707.

48. Ferraro A, et al. Expansion of Th17 cells and functional defects in T regulatory cells are key features of the pancreatic lymph nodes in patients with type 1 diabetes. Diabetes. 2011;60(11):2903-2913.

49. Gao X, et al. Adjuvant treatment suppresses IL-17 production by T cell-independent myeloid sources in nonobese diabetic mice. Mol Immunol. 2010;47(14):2397-2404.

50. Emamaullee JA, et al. Inhibition of Th17 cells regulates autoimmune diabetes in NOD mice. Diabetes. 2009;58(6):1302-1311.

51. Jain $\mathrm{R}$, et al. Innocuous IFN $\gamma$ induced by adjuvant-free antigen restores normoglycemia in NOD mice through inhibition of IL-17 production. J Exp Med. 2008;205(1):207-218.

52. Reinert-Hartwall L, et al. Th1/Th17 plasticity is a marker of advanced $\beta$ cell autoimmunity and impaired glucose tolerance in humans. J Immunol. 2015;194(1):68-75.

53. Kuriya G, et al. Double deficiency in IL-17 and IFN- $\gamma$ signalling significantly suppresses the development of diabetes in the NOD mouse. Diabetologia. 2013;56(8):1773-1780.

54. Bertin-Maghit S, et al. Interleukin-1 $\beta$ produced in response to islet autoantigen presentation differentiates T-helper 17 cells at the expense of regulatory T-cells: Implications for the timing of tolerizing immunotherapy. Diabetes. 2011;60(1):248-257.

55. Li CR, Mueller EE, Bradley LM. Islet antigen-specific Th17 cells can induce TNF- $\alpha$-dependent autoimmune diabetes. J Immunol. 2014;192(4):1425-1432.

56. Kriegel MA, Sefik E, Hill JA, Wu HJ, Benoist C, Mathis D. Naturally transmitted segmented filamentous bacteria segregate with diabetes protection in nonobese diabetic mice. Proc Natl Acad Sci U S A. 2011;108(28):11548-11553.

57. Tse HM, et al. NADPH oxidase deficiency regulates Th lineage commitment and modulates autoimmunity. J Immunol. 2010;185(9):5247-5258

58. Nikoopour E, et al. Th17 polarized cells from nonobese diabetic mice following mycobacterial adjuvant immunotherapy delay type 1 diabetes. J Immunol. 2010;184(9):4779-4788

59. Lau K, et al. Inhibition of type 1 diabetes correlated to a Lactobacillus johnsonii N6.2-mediated Th17 bias. J Immunol. 2011;186(6):3538-3546.

60. Carrascal J, et al. B-cell anergy induces a Th17 shift in a novel B lymphocyte transgenic NOD mouse model, the 116C-NOD mouse. Eur J Immunol. 2016;46(3):593-608.

61. Ishigame H, et al. Excessive Th1 responses due to the absence of TGF- $\beta$ signaling cause autoimmune diabetes and dysregulated Treg cell homeostasis. Proc Natl Acad Sci U S A. 2013;110(17):6961-6966.

62. Joseph J, Bittner S, Kaiser FM, Wiendl H, Kissler S. IL-17 silencing does not protect nonobese diabetic mice from autoimmune diabetes. J Immunol. 2012;188(1):216-221.

63. Liu SM, et al. Differential IL-21 signaling in APCs leads to disparate Th17 differentiation in diabetes-susceptible NOD and diabetes-resistant NOD.Idd3 mice. J Clin Invest. 2011;121(11):4303-4310.

64. Ramesh R, et al. Pro-inflammatory human Th17 cells selectively express P-glycoprotein and are refractory to glucocorticoids. $J$ Exp Med. 2014;211(1):89-104.

65. Bresson D, et al. Anti-CD3 and nasal proinsulin combination therapy enhances remission from recent-onset autoimmune diabetes by inducing Tregs. J Clin Invest. 2006;116(5):1371-1381.

66. Baas MC, et al. Combining autologous dendritic cell therapy with CD3 antibodies promotes regulatory $\mathrm{T}$ cells and permanent islet allograft acceptance. J Immunol. 2014;193(9):4696-4703.

67. Hu C, Ding H, Zhang X, Wong FS, Wen L. Combination treatment with anti-CD20 and oral anti-CD3 prevents and reverses autoimmune diabetes. Diabetes. 2013;62(8):2849-2858.

68. Ablamunits V, et al. Synergistic reversal of type 1 diabetes in NOD mice with anti-CD3 and interleukin-1 blockade: evidence of improved immune regulation. Diabetes. 2012;61(1):145-154.

69. Moran A, et al. Interleukin-1 antagonism in type 1 diabetes of recent onset: two multicentre, randomised, double-blind, placebo-controlled trials. Lancet. 2013;381(9881):1905-1915.

70. Schiering C, et al. The alarmin IL-33 promotes regulatory T-cell function in the intestine. Nature. 2014;513(7519):564-568

71. Vasanthakumar A, et al. The transcriptional regulators IRF4, BATF and IL-33 orchestrate development and maintenance of adipose tissue-resident regulatory T cells. Nat Immunol. 2015;16(3):276-285.

72. Kolodin D, et al. Antigen- and cytokine-driven accumulation of regulatory T cells in visceral adipose tissue of lean mice. Cell Metab. 2015;21(4):543-557.

73. Meghnem D, et al. Cutting Edge: differential fine-tuning of IL-2- and IL-15-dependent functions by targeting their common IL-2/15R $\beta / \gamma c$ receptor. J Immunol. 2017;198(12):4563-4568. 
74. Boyman O, Kovar M, Rubinstein MP, Surh CD, Sprent J. Selective stimulation of T cell subsets with antibody-cytokine immune complexes. Science. 2006;311(5769):1924-1927.

75. Levin AM, et al. Exploiting a natural conformational switch to engineer an interleukin-2 'superkine'. Nature. 2012;484(7395):529-533.

76. Hill JA, et al. Foxp3 transcription-factor-dependent and -independent regulation of the regulatory T cell transcriptional signature. Immunity. 2007;27(5):786-800.

77. Fu W, et al. Epigenetic modulation of type-1 diabetes via a dual effect on pancreatic macrophages and $\beta$ cells. Elife. 2014;3:e04631.

78. Mortier E, et al. Soluble interleukin-15 receptor alpha (IL-15R $\alpha$ )-sushi as a selective and potent agonist of IL-15 action through IL-15R $\beta / \gamma$. Hyperagonist IL-15 x IL-15R $\alpha$ fusion proteins. J Biol Chem. 2006;281(3):1612-1619.

79. Stauber DJ, Debler EW, Horton PA, Smith KA, Wilson IA. Crystal structure of the IL-2 signaling complex: paradigm for a heterotrimeric cytokine receptor. Proc Natl Acad Sci U S A. 2006;103(8):2788-2793.

80. Tanaka T, Kitamura F, Nagasaka Y, Kuida K, Suwa H, Miyasaka M. Selective long-term elimination of natural killer cells in vivo by an anti-interleukin 2 receptor $\beta$ chain monoclonal antibody in mice. J Exp Med. 1993;178(3):1103-1107.

81. Arduin E, et al. Highly reduced binding to high and low affinity mouse Fc $\gamma$ receptors by L234A/L235A and N297A Fc mutations engineered into mouse IgG2a. Mol Immunol. 2015;63(2):456-463.

82. Xu D, et al. In vitro characterization of five humanized OKT3 effector function variant antibodies. Cell Immunol. 2000;200(1):16-26.

83. Waldmann TA, et al. Phase 1 trial of IL-15 trans presentation blockade using humanized Mik $\beta 1 \mathrm{mAb}$ in patients with T-cell large granular lymphocytic leukemia. Blood. 2013;121(3):476-484

84. Roederer M. Interpretation of cellular proliferation data: avoid the panglossian. Cytometry A. 2011;79(2):95-101. 